\title{
Turbulent shear-layer mixing: growth-rate compressibility scaling
}

\author{
By M. D. SLESSOR ${ }^{1}$, M. ZHUANG ZND P. E. DIMOTAKIS $^{3}$ \\ ${ }^{1}$ Allied Signal AMM, 1349 Moffett Park Drive, Sunnyvale, CA 94089, USA \\ ${ }^{2}$ Mechanical Engineering, Michigan State University, East Lansing, MI 48824, USA \\ ${ }^{3}$ Graduate Aeronautical Laboratories, California Institute of Technology, \\ Pasadena, CA 91125, USA
}

(Received 29 June 1998 and in revised form 11 August 1999)

A new shear-layer growth-rate compressibility-scaling parameter is proposed as an alternative to the total convective Mach number, $M_{c}$. This parameter derives from considerations of compressibility as a means of kinetic-to-thermal-energy conversion and can be significantly different from $M_{c}$ for flows with far-from-unity free-streamdensity and speed-of-sound ratios. Experimentally observed growth rates are wellrepresented by the new scaling.

\section{Introduction}

In the context of shear-layer mixing, the growth rate, $\delta^{\prime}(x)$, where $\delta(x)$ is the local transverse extent of the turbulent region, is important. It sets an upper bound for the amount of molecular mixing one may expect under a particular set of flow conditions (Dimotakis 1991a). For linearly growing shear layers, we have

$$
\delta^{\prime}(x) \simeq \frac{\delta(x)-\delta\left(x_{0}\right)}{x-x_{0}} \simeq \frac{\delta(x)}{x} .
$$

The latter equality holds if $\delta\left(x_{0}\right) \ll \delta(x)$ and $x_{0} \ll x$, in which case the ratio $\delta(x) / x$ may be used as an estimate of the growth rate. It is often employed as such in interpreting experimental data.

Compressibility effects on the turbulent shear-layer growth rate, $\delta^{\prime}(x)$, are often scaled by the total convective Mach number (Papamoschou 1989),

$$
M_{c} \equiv \frac{U_{1}-U_{2}}{a_{1}+a_{2}}=\frac{1}{1+\left(a_{2} / a_{1}\right)}\left(\frac{\Delta U}{a_{1}}\right),
$$

where $U_{i}$ and $a_{i}$ are the flow speed and speed of sound of the $i$ th free stream, respectively. In particular, compressibility has been assumed to act independently of free-stream velocity and density ratios, i.e.

$$
\frac{\delta^{\prime}}{\delta_{0}^{\prime}}\left(M_{c}\right) \neq \mathrm{fn}\left(r=\frac{U_{2}}{U_{1}}, s=\frac{\rho_{2}}{\rho_{1}}\right),
$$

where $\delta_{0}^{\prime}(x)$ is the growth rate of a shear layer in the limit of zero (convective) Mach number, at the same velocity and density ratio, i.e.

$$
\delta_{0}^{\prime}(x ; r, s) \equiv \frac{\partial}{\partial x} \delta\left(x ; r, s, M_{c} \rightarrow 0\right) .
$$


This proposal was corroborated by the results of linear-stability analysis by Ragab \& Wu $(1987,1988,1989)$, who correlated shear-layer growth with the most-unstablemode eigenvalue, i.e.

$$
-\alpha_{i, \max } \propto \delta^{\prime}(x)
$$

over a limited free-stream-density-ratio range. They concluded that the convective Mach numbers proposed by Papamoschou \& Roshko (1986, 1988),

$$
M_{c, 1}=\frac{U_{1}-U_{c}}{a_{1}} \text { and } M_{c, 2}=\frac{U_{c}-U_{2}}{a_{2}},
$$

where $U_{c}$ is an appropriate convection velocity of turbulent structures, provide useful compressibility-scaling parameters and correlate shear-layer growth rate and compressibility. Sandham \& Reynolds (1989) proposed that shear-layer growth could be modelled in terms of the most-unstable eigenvalue for a particular shear-layer base-flow profile. Further linear-stability analyses by Zhuang, Dimotakis \& Kubota (1990a), Zhuang, Kubota \& Dimotakis (1990b) and Zhuang (1990), for a range of flow parameters and geometry, provided additional support for this proposal.

An issue in the parametrizations above is the choice of the convection velocity, $U_{c}$, which is not unique. For nearly matched free-stream specific-heat ratios, i.e. for $\gamma_{1} \simeq \gamma_{2}$, equation (5) yields $M_{c, 1} \simeq M_{c, 2} \simeq M_{c}$, if the convection velocity is estimated using the isentropic pressure-recovery model of Papamoschou \& Roshko (1988). Other choices have also been argued for. They include the phase speed of the (linearly) most-unstable mode (Ragab \& Wu 1989), its phase speed at a point in its (linear) evolution corresponding to neutral stability (Sandham \& Reynolds 1989), its phase speed as modified by reflected-/resonant-wave systems in bounded shear layers (Tam \& Hu 1989; Zhuang et al. 1990b), or, for more complicated mixinglayer flows, e.g. shear layers with wake components, the phase speed of the most unstable mode, in each case (Zhuang \& Dimotakis 1995). Finally, for supersonic shear layers that can support shocks between convected turbulent structures and the respective free streams (Papamoschou 1989; Dimotakis 1989, 1991a, $b$; Papamoschou \& Bunyajitradula 1996), models for the convective speed of turbulent structures in the presence of shocks (Dimotakis 1991b) provide yet another alternative.

Estimates for $\delta_{0}^{\prime}(x)=\delta^{\prime}\left(x ; M_{c} \rightarrow 0\right)$, the incompressible-flow growth used to normalize the compressible-flow growth rate, have typically relied on the Brown (1974) temporal-growth model, or the Dimotakis (1986) spatial-growth model, i.e.

$$
\delta_{0}^{\prime}(x ; r, s) \simeq C_{\delta} \frac{(1-r)\left(1+s^{1 / 2}\right)}{2\left(1+s^{1 / 2} r\right)}\left\{1-\frac{\left(1-s^{1 / 2}\right) /\left(1+s^{1 / 2}\right)}{1+2.9(1+r) /(1-r)}\right\},
$$

where $C_{\delta}$ is assumed to be a constant (independent of $r$ and $s$ ). The Brown (1974) temporal-growth model yields a similar estimate, with the exception of the term in braces, which stems from spatial-growth asymmetries.

The Brown \& Roshko (1974) experimental data provide a good assessment of density-ratio effects, with values for $\delta_{0}^{\prime}$ reported for $1 / 7 \leqslant s \leqslant 7$ and $0 \leqslant r \leqslant \sqrt{1 / 7}$. The velocity- and density-ratio dependence prediction of the two models (filled and open symbols) are compared and tested against the Brown-Roshko growth-rate data in figure 1 . The model predictions were computed as $\delta_{0}^{\prime} / C_{\delta}$ (cf. equation (6)), i.e. assuming no particular value for $C_{\delta}$. The model predictions are seen to be close to each other and to provide reasonably good estimates of incompressible shear-layer growth, with no systematic deviation from the experimental values as a function of the free-stream density ratio, $s$. The straight line in figure 1 is a least-squares fit 


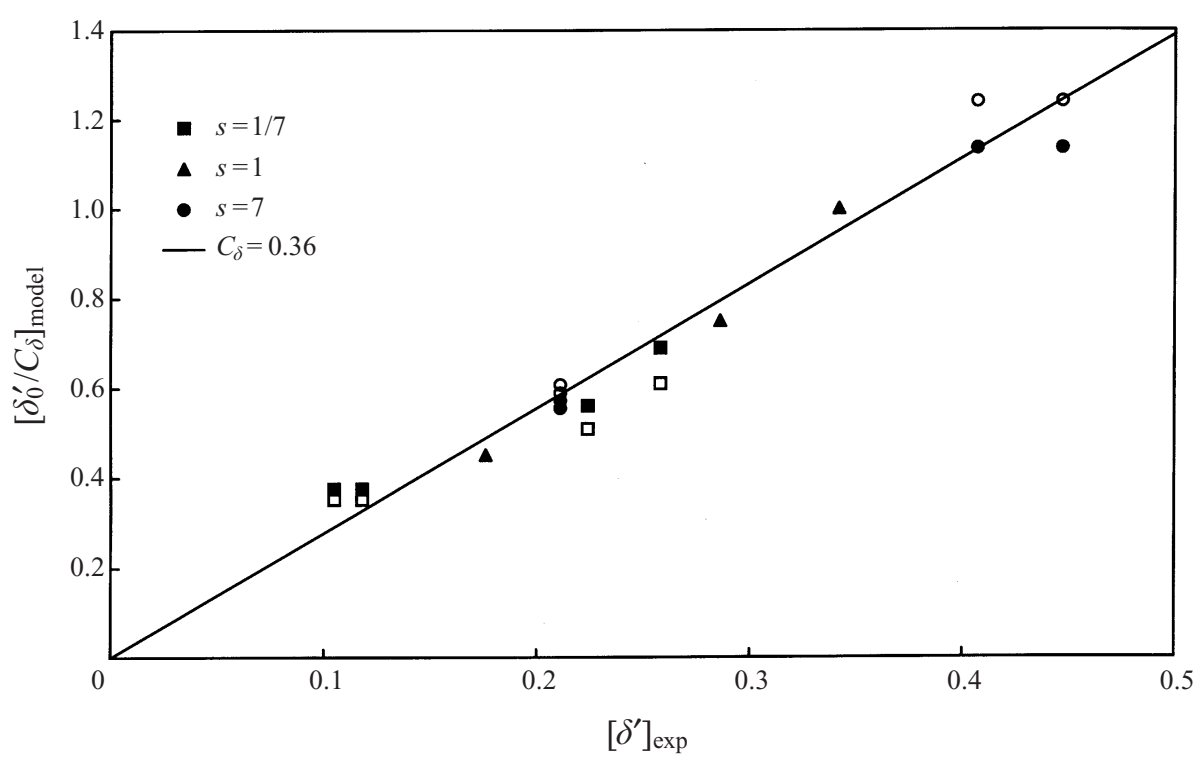

FIGURE 1. Brown (1974, filled symbols) and Dimotakis (1986, open symbols), shear-layer-growth models vs. incompressible data of Brown \& Roshko (1974). Note that model estimates coincide for $s=1$ (cf. equation (6)).

(through the origin), yielding a value of the growth constant of $C_{\delta}=0.36$ for this data set.

Although experimental incompressible-flow growth-rate data are in reasonable accord with equation (6), there is substantial variance in the inferred values for the growth-rate constant, i.e.

$$
0.25 \lesssim C_{\delta} \lesssim 0.45 \text {, }
$$

with arguments and evidence that this is dependent on facility, inflow conditions, and other flow details (Dimotakis 1991a), with further difficulties because of differences in the definitions of shear-layer thickness employed by different investigators (cf. Papamoschou 1986). Such reasons could also be held partly responsible for the considerable variance in compressible-flow growth-rate data.

In an attempt to reconcile differences between different experiments, $C_{\delta}$ can be evaluated separately for each individual data set, as also suggested by Lu \& Lele (1994). The resulting scaled growth-rate data, $\delta^{\prime} / \delta_{0}^{\prime}$, are plotted in figure 2 vs. total convective Mach number, $M_{c}$ (equation (2)). The procedure and resulting values for $C_{\delta}$ are documented in the Appendix. As can be seen, despite adjustments to the growth-rate constant, $C_{\delta}$, i.e. even after allowing for possible facility- and experimentdependent variations in growth rate, the scaling suggested by equation (3) does not provide a good collapse, with differences between experimental growth rates and inferred mean behaviour vs. $M_{c}$ as large as a factor of two. In particular, low growth rates are documented in flows with extreme free-stream-density ratios, e.g. $s \simeq 9.2$ for Case 6 in Papamoschou \& Roshko (1988) and $s \simeq 0.058$ for Case 9 in Hall, Dimotakis \& Rosemann (1993), the points in each data set farthest below the notional curve through the data in figure 2 . They will be discussed further, below.

The systematic deviation for far-from-unity free-stream density ratios suggests that this may be attributable either to an incorrect normalization, as suggested by Lu \& 


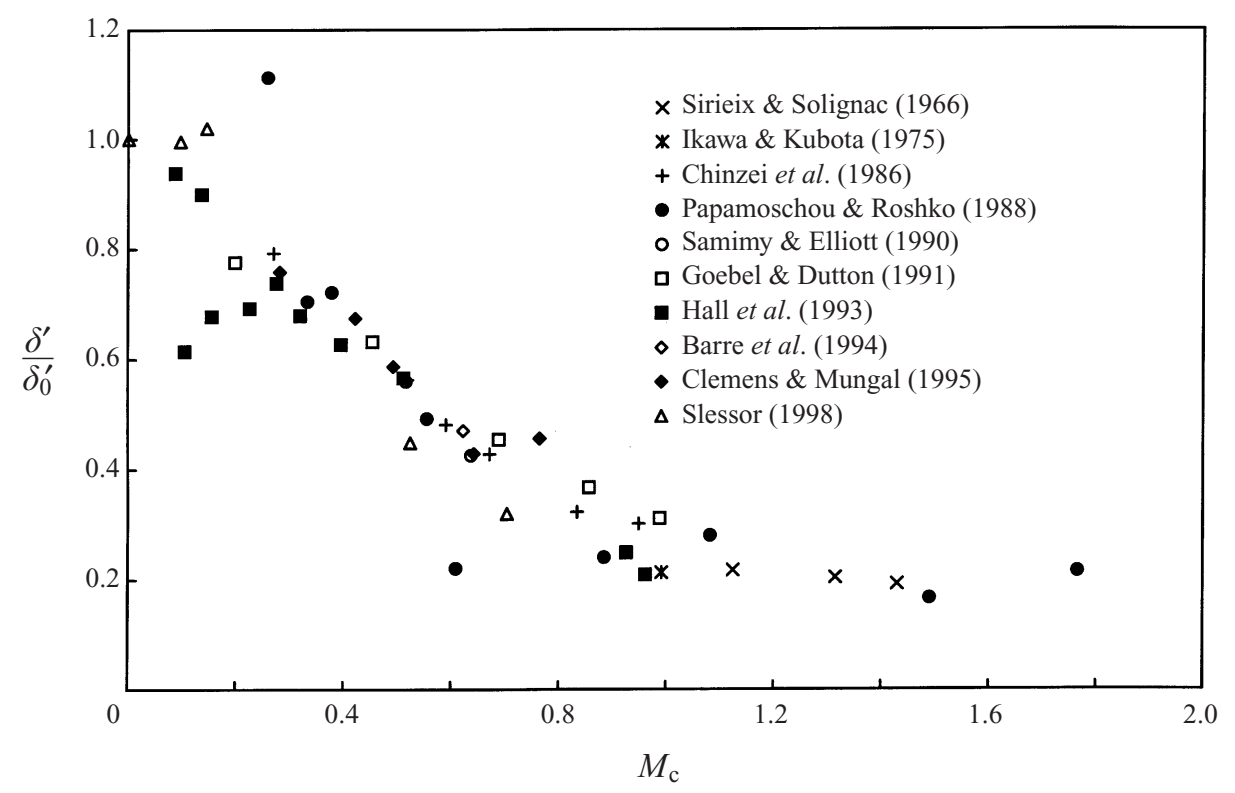

FIGURE 2. Normalized compressible shear-layer growth rates vs. total convective Mach number, $M_{c}$ (equation (2)). Incompressible-flow estimates, $\delta_{0}^{\prime}$, provided by equation (6).

Lele (1994), or to improper compressibility scaling. These possibilities will also be discussed below.

\section{Incompressible shear-layer growth rates}

Lu \& Lele (1994) reported improved scaling of compressible shear-layer growth rates in terms of the most-unstable eigenvalue for incompressible flow, $\left(-\alpha_{i, \max }\right)_{0}$, as derived from linear-stability analysis. In particular, they proposed that

$$
\frac{\delta^{\prime}}{\left(-\alpha_{i, \max }\right)_{0}} \approx \mathrm{fn}\left(M_{c}\right) \text {. }
$$

In reference to equation (3), this prescription assumes that the most-unstable eigenvalues are proportional to the incompressible shear-layer growth rate, $\delta_{0}^{\prime}$. Figure 5 of Lu \& Lele (1994), for example, supports the proposal in equation (4). When combined with equation (4), this yields (cf. equation (8))

$$
\frac{-\alpha_{i, \max }}{\left(-\alpha_{i, \max }\right)_{0}} \approx \mathrm{fn}\left(M_{c}\right) \quad \text { or } \quad \frac{\delta^{\prime}}{\left(-\alpha_{i, \max }\right)_{0}} \approx \mathrm{fn}\left(M_{c}\right),
$$

as was also assumed in the comparisons with experimentally observed growth rates by the linear stability analyses cited above. However, successful scaling of compressibility in terms of equation (9) does not imply correct predictions of growth rate.

Both compressibility scaling and the incompressible-flow growth rate must be correctly accounted for that to be achieved. Successful scaled-data collapse in terms of the ratio of most-unstable eigenvalues is insufficient, of itself, in validating linearstability analysis as a growth-rate predictor.

It is unresolved whether incompressible-flow growth rates, $\delta_{0}^{\prime}$, are correlated with the most-unstable eigenvalue, $\left(-\alpha_{i, \max }\right)_{0}$, over the shear-layer flow conditions that 


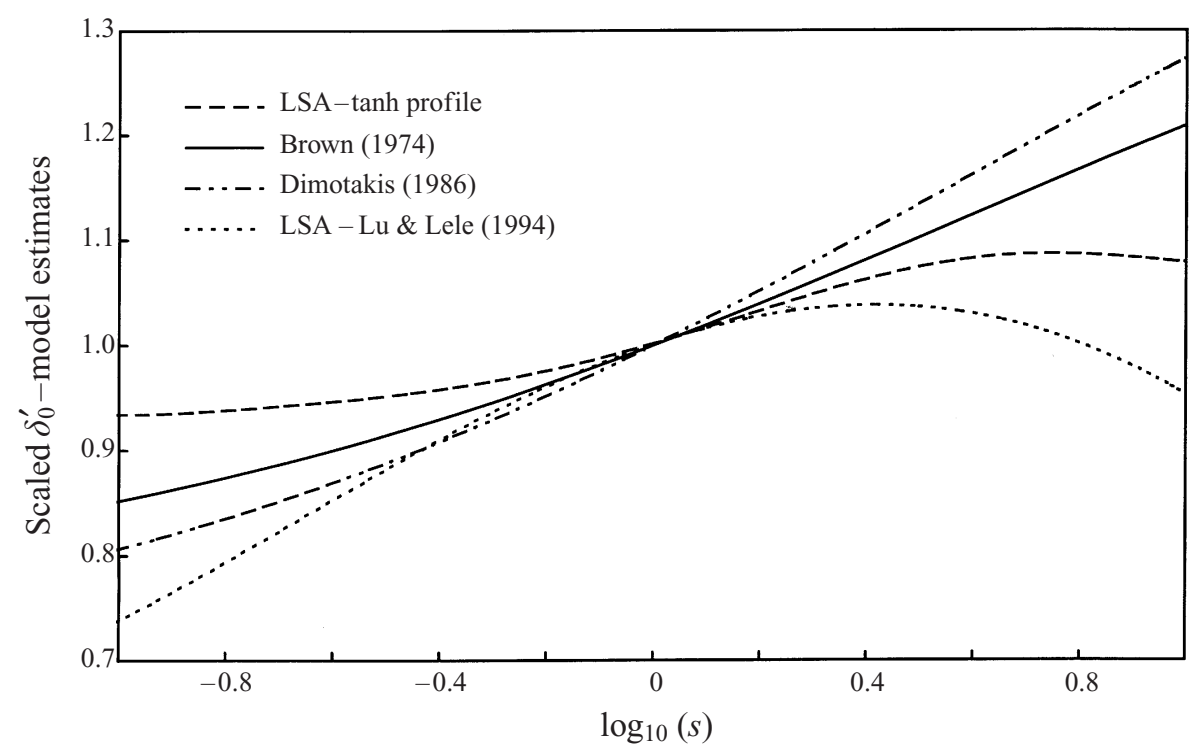

FIGURE 3. Comparison of Brown (1974), Dimotakis (1986), and linear-stability-analysis (LSA) model estimates, normalized at $s=1$, computed for incompressible shear-layer growth at $r=1 / 2$.

must be accounted for. This is especially true at extreme values of the free-streamdensity ratio, a regime where, as noted above, the main difficulties lie and where the utility of linear-stability analysis for shear layers has not been as well documented. Interestingly, figure 5 in $\mathrm{Lu} \&$ Lele (1994) suggests a poor correlation with the freestream density-ratio dependence for incompressible flows (cf. Cases 10 and 11 of Hall et al. 1993).

The linear-stability estimates of $\mathrm{Lu} \&$ Lele (1994) were provided for a velocity ratio of $r=1 / 2$, which, unfortunately, does not correspond to shear-layer flows for which experimental data are available. Consequently, a direct test of their proposal against experimental data is not possible. Accordingly, a comparison of the candidate incompressible growth-rate estimates is presented in figure 3 , for $r=1 / 2$, relying on the comparison between the Brown and Dimotakis structure-based growth models and the experimental data (figure 1) to assess model growth-rate predictions vs. the free-stream density ratio, $s$. The Lu \& Lele linear-stability estimates can be seen to offer qualitatively different predictions at extreme density ratios, with much-lower growth rates than either of the structure-based models, a behaviour required for the collapse reported by Lu \& Lele, if the parametrization in terms of total convective Mach number, $M_{c}$, is retained.

As has been noted, linear-stability analysis can be rather sensitive to the base-flow profile(s) assumed (e.g. Sandham \& Reynolds 1989; Kozusko et al. 1996; Peroomian \& Kelly 1996; Kennedy \& Chen 1998). Lu \& Lele employed a set of velocity profiles based on a solution to the laminar, thin-shear-layer equations. The two free streams were assumed to have the same composition, with free-stream density-ratio effects simulated in terms of different free-stream temperatures. Their representation should be compared with the He/Ar (Case 9) flow of Hall et al. (1993), for example. Alternatively, as can be seen in figure 3, a hyperbolic-tangent velocity profile, often employed as a shear-layer base flow, produces linear-stability estimates for small free-stream-density ratios, $s=\rho_{2} / \rho_{1} \ll 1$, that exceed those of the structure-based 
models. When used to normalize compressible-flow growth rates for extreme freestream-density ratios, this would result in estimated normalized growth rates that would be lower yet than those in figure 2 .

\section{Proposed compressibility scaling}

Turbulent velocity fluctuations and their correlations are coupled to shear-layer growth, as can be inferred from the Reynolds-averaged momentum equation. Experimental confirmation of this association includes decreased (r.m.s.) velocity fluctuations with reduced shear-layer growth rates (Goebel et al. 1990; Geobel \& Dutton 1991), and diminished values of $\overline{u^{\prime} v^{\prime}}$ at compressible-flow conditions (Samimy \& Elliott 1990). This relationship has been shown in terms of the turbulent kinetic energy (Sarkar 1995) and Reynolds-stress production (Vreman, Sandham \& Luo 1996; Freund, Moin \& Lele 1997). These authors also argue for a growth-rate suppression mechanism attributable to inviscid energy-exchange mechanisms and not to increased viscous (dilatational) dissipation, as had been suggested by Zeman (1990). Recall that the experimentally observed linear mean growth rates indicate no growth-rate dependence on the local Reynolds number, which increases linearly with downstream distance, as would be expected if viscous mechanisms were important.

To motivate the present proposal, we note that, in the most general terms, compressibility couples kinetic and thermal energy. For example, for the kinetic-to-thermal energy (enthalpy) ratio, we have (for a perfect gas)

$$
\frac{u^{2}}{2 h} \simeq \frac{\gamma-1}{2} M^{2},
$$

where $u$ is the velocity, $h$ the specific enthalpy, $\gamma$ the specific-heat ratio, and $M$ the Mach number. This scaling assumes that the energy conversion is adiabatic, but not necessarily isentropic, and is ubiquitous in all forms of the dimensionless energy equation (e.g. Lagerstrom 1964), even in its full unsteady form. Kinetic-to-thermal energy conversion becomes increasingly important with increasing (relative) Mach number and flow compressibility, with velocity fluctuations increasingly coupled to thermal-energy (enthalpy) fluctuations.

In a shear layer, the free-stream-density ratio, $s=\rho_{2} / \rho_{1}$, is related to the speed-ofsound ratio, i.e. (for a perfect gas)

$$
\frac{a_{2}}{a_{1}}=\sqrt{\frac{\gamma_{2} p_{2} / \rho_{2}}{\gamma_{1} p_{1} / \rho_{1}}} \simeq \sqrt{\frac{\gamma_{2} / \gamma_{1}}{s}},
$$

where the second equality holds for static-pressure-matched free streams, i.e. $p_{2} \simeq p_{1}$, as is approximately the case for (low-displacement) two-dimensional, planar shear layers. A shear layer with a small free-stream-density ratio (e.g. Case 9 of Hall et al. 1993) has a correspondingly large speed-of-sound ratio, as indicated by equation (11). In far-from-unity-s flows, scaling flow compressibility by $M_{c}$ will be dominated by the large speed-of-sound ratio (cf. equation (2)). Given the observed propensity of these flows to exhibit asymmetric large-scale-structure convection speeds (Papamoschou 1989; Dimotakis 1989, 1991a, b; Papamoschou \& Bunyajitradula 1996), resulting in correspondingly large actual convective-frame Mach numbers, such scaling is likely to misrepresent flow compressibility.

Forming the group suggested by equation (10) with the (frame-independent) freestream velocity difference, $\Delta U$ (cf. equation (5)), and accepting the larger of the two 


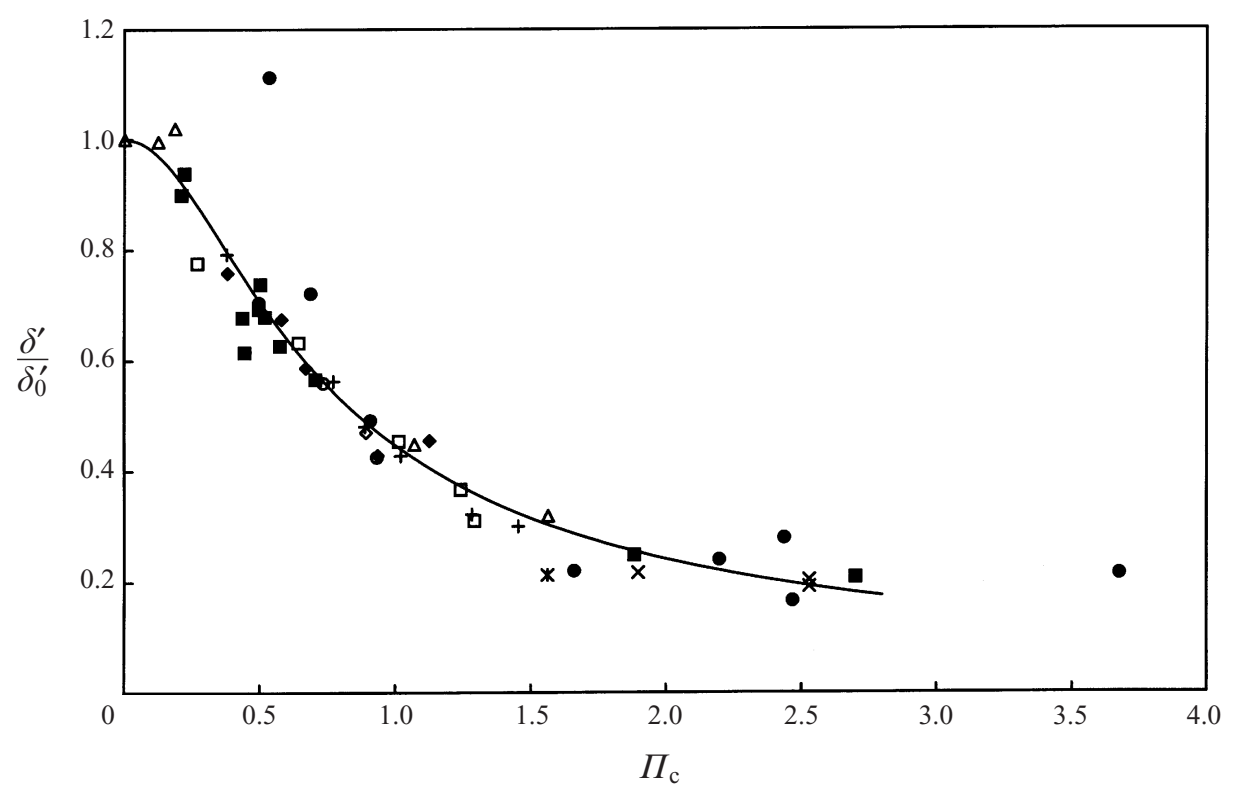

FIGURE 4. Normalized compressible shear-layer growth rates (as in figure 2), vs. proposed compressibility parameter $\Pi_{c}$ (equation (12)). Solid curve given by equation (13). Legend as figure 2 .

choices as an ansatz, yields an alternative compressibility parameter,

$$
\Pi_{c}=\max _{i}\left[\frac{\sqrt{\gamma_{i}-1}}{a_{i}}\right] \Delta U .
$$

The experimentally measured scaled growth rates are plotted in figure 4 , vs. $\Pi_{c}$. The plot should be compared with that in figure 2 and can be seen to exhibit a much-improved collapse. The fitted smooth curve through the data is given by

$$
\frac{\delta^{\prime}}{\delta_{0}^{\prime}}\left(\Pi_{c}\right)=\left(1+\alpha \Pi_{c}^{2}\right)^{-\beta}, \quad \text { with } \alpha \simeq 4, \beta \simeq 0.5,
$$

and is not extended to high-compressibility conditions.

The systematic deviations in flows with extreme density/speed-of-sound ratios can be seen to be largely accounted for. Of course, all the complex dynamics that give rise to compressible shear-layer growth cannot be represented by a single parameter and while the collapse is improved, it is not perfect. On the other hand, in view of the substantial variance in the observed growth of shear layers with ostensibly identical flow parameters (recall equation (7) and related discussion), a better collapse may well not be possible.

Considering that many flows among the data compiled are characterized by thermodynamically similar free-stream fluids, e.g. the air/air experiments of Chinzei et al. (1986), corresponding to near-unity $a_{2} / a_{1}$ ratios and matched $\gamma_{i}$, it is not surprising that the previous scaling of normalized growth-rate data appeared adequate. In particular, since, at these conditions,

$$
\Pi_{c}\left(a_{2}=a_{1}, \gamma_{2}=\gamma_{1}=\gamma\right)=2 \sqrt{\gamma-1} M_{c},
$$

the previous collapse of the growth-rate data for such flows will be preserved when 


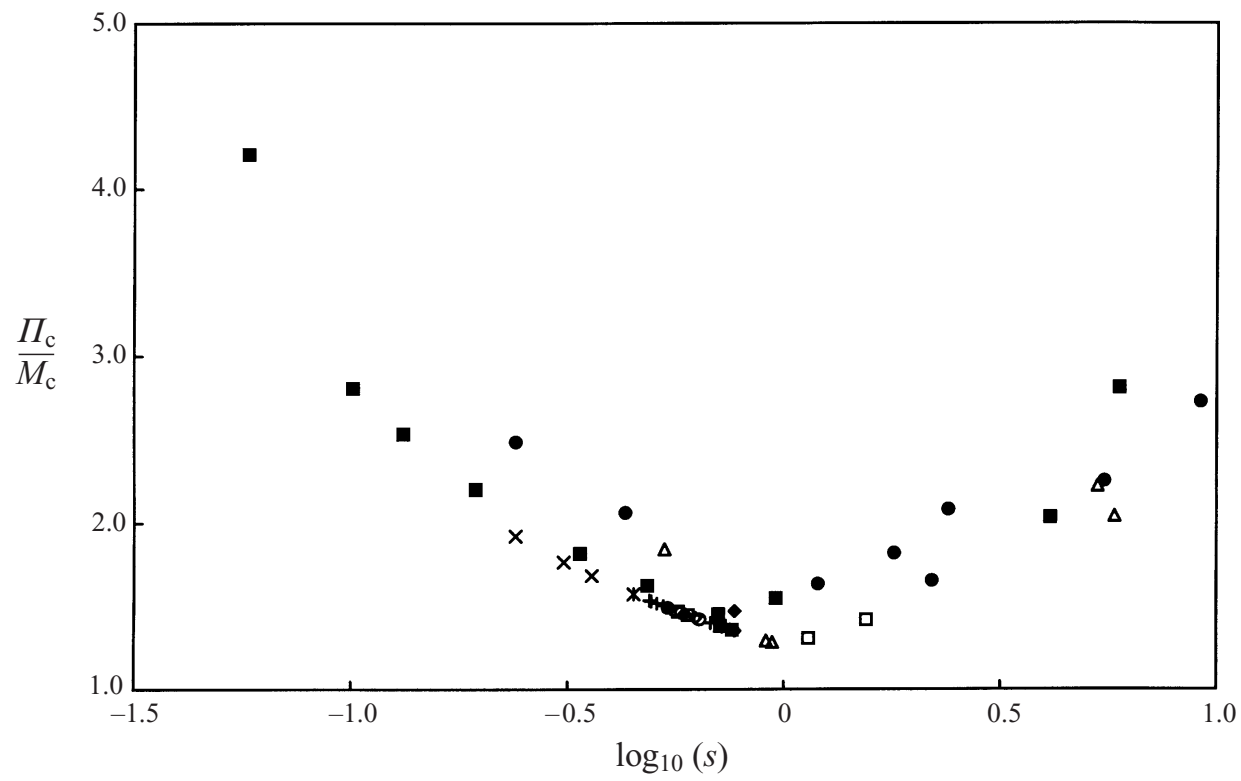

FIgURE 5. The ratio $\Pi_{c} / M_{c}$, vs. $\log _{10}\left(s=\rho_{2} / \rho_{1}\right)$, computed for the data compiled in figures 2 and 4 . Legend as figure 2.

scaled in terms of the new parameter, $\Pi_{c}$, as borne out by figure 4 . Conversely, for shear-layer flows for which the free-stream-density ratio, $s=\rho_{2} / \rho_{1}$ is far from unity, there are large differences between $\Pi_{c}$ and $M_{c}$. This is illustrated in figure 5, that plots the ratio $\Pi_{c} / M_{c}$ vs. $\log _{10}(s)$, for all the data compiled in the previous plots. The low growth rates documented for the flows with extreme free-stream-density ratios, e.g. $s \simeq 9.2$ for Case 6 in Papamoschou \& Roshko (1988) and $s \simeq 0.058$ for Case 9 in Hall et al. (1993), discussed above, are the outliers in figure 5, with a correspondingly large ratio between $\Pi_{c}$ and $M_{c}$. The proposed scaling can then be seen as responsible for bringing them in closer compliance with a more-uniform similarity behaviour.

Alternative compressibility-scaling parameters were also considered. It could be argued, for example, that actual convective-frame Mach numbers (equation (5)), with a convective-frame speed, $U_{c}$, computed in a way that reflects the asymmetry in $M_{c, 1}$ and $M_{c, 2}$ discussed above, might serve as good candidates. The large asymmetries in the two convective Mach numbers yield values of one of the two close to the proposed $\Pi_{c}$ compressibility parameter, e.g. with convective speeds computed with the shock-formation model (Dimotakis 1991b), $\max _{i}\left\{M_{c, i}\right\} \approx \mathrm{fn}\left(\gamma_{i}\right) \Pi_{c}$ (cf. equations (5) and (12)). While such a scheme captures some of the same physics and yields improved scaling relative to that in figure 2 , it does not do as well.

\section{Conclusions}

A new shear-layer growth-rate compressibility parameter is proposed, motivated by available shear-layer growth-rate data and a view of compressibility as an energytransformation mechanism. This parameter differs from the previously employed convective or total Mach numbers, especially at extreme free-stream-density ratios, where future, high-speed air-breathing-propulsion vehicles are expected to operate, and provides a good collapse of available shear-layer growth rates.

Entrainment, which is responsible for growth, i.e. the means by which free-stream 


$\begin{array}{llclccc} & M_{c, \text { ref }} & f\left(M_{c, \text { ref }}\right) & \delta_{0}^{\prime} / C_{\delta} & \delta^{\prime}\left(M_{c, \text { ref }}\right) & C_{\delta} & C_{\delta, 0} \\ \text { Sirieix \& Solignac } & 1.13 & 0.218 & 1.50 & 0.0345 & 0.212 & 0.280 \\ \text { Ikawa \& Kubota } & 0.991 & 0.242 & 1.59 & 0.064 & 0.378 & 0.210 \\ \text { Chinzei et al. } & 0.591 & 0.481 & 0.805 & 0.045 & 0.232 & 0.280 \\ \text { Papamoschou \& Roshko } & 0.555 & 0.517 & 0.400 & 0.026 & 0.264 & 0.280 \\ \text { Samimy \& Elliott } & 0.517 & 0.559 & 0.881 & 0.093 & 0.378 & 0.210 \\ \text { Goebel \& Dutton } & 0.453 & 0.632 & 0.570 & 0.038 & 0.210 & 0.165 \\ \text { Hall } \text { et al. } & 0.511 & 0.565 & 1.16 & 0.114 & 0.348 & 0.340 \\ \text { Barre } \text { et al. } & 0.623 & 0.450 & 1.19 & 0.0338 & 0.121 & 0.121 \\ \text { Clemens \& Mungal } & 0.493 & 0.586 & 1.05 & 0.100 & 0.326 & 0.340\end{array}$

TABLE $1 . C_{\delta}$ determination. See text for commentary on data sources.

fluid enters in the dynamics of turbulent structures, should be viewed as occurring in the frame of those structures. In that sense, the convective Mach number(s) employed previously explicitly relied on a posited frame for entrainment, which differs in various proposals, as discussed in the Introduction. The improvement in scaling afforded by the proposed parameter suggests that compressibility effects in high-speed shear layers are dominated by flow modes that are not peculiar to a particular frame. This is, of course, also the case for the total convective Mach number (equation (2)), even though, as argued above, this under-represents compressibility effects in shear layers formed between free-stream fluids with a far-from-unity density ratio. In high-Reynolds-number flows, the variance of possible flow modes and turbulent structures is very high, with a range of local convection speeds (celerities) that can also be high. The improved scaling stemming from the proposed parameter implies that it is the maximum possible compressibility of flow-structure modes (max of two possible choices in equation (12)) that can live across the full shear-layer transverse extent (i.e. full $\Delta U$ ), that scales compressibility effects.

This work and the recent experiments cited (Slessor 1998) were supported by the Air Force Office of Scientific Research, Grants F49620-93-1-0338, F49620-94-1-0353, and F49620-98-1-0052. We would like to acknowledge the help of Earl Dahl in the execution of the recent experiments and the suggestion by one of the referees that the relation between $\Pi_{c}$ and $M_{c}$ should be depicted, leading to figure 5 .

\section{Appendix}

An attempt was made to reconcile the data from various experiments by allowing for different growth-rate constants, $C_{\delta}$, for each data set.

Most data sets include measurements near $M_{c}=0.5$. In this Mach number regime, the empirical correlation (Dimotakis 1991a)

$$
f\left(M_{c}\right)=0.8 \mathrm{e}^{-3 M_{c}^{2}}+0.2
$$

was employed as a local interpolator of the normalized growth rate, $\delta^{\prime}\left(M_{c}\right) / \delta_{0}^{\prime}$, in the vicinity of $M_{c}=0.5$. It was used to estimate the growth constant,

$$
C_{\delta} \simeq \frac{\delta^{\prime}\left(M_{c, \text { ref }}\right)}{f\left(M_{c, \text { ref }}\right) \delta_{0}^{\prime} / C_{\delta}},
$$

for each data set, where $\delta_{0}^{\prime} / C_{\delta}$ is computed using equation $(6), \delta^{\prime}\left(M_{c, \text { ref }}\right)$ is the 
measured growth rate referenced to $M_{c}=M_{c, \text { ref }}$, and $f\left(M_{c, \text { ref }}\right)$ is computed using (A 1). This procedure yields the values for $C_{\delta}$ listed in table 1 , where the $C_{\delta, 0}$ are values suggested in (or deduced from) the original publications. The results are relatively insensitive to the actual correlation form assumed (A 1). The values of $C_{\delta}$ are as required in equation (6). Note that these differ by a factor of 2 from those used by investigators that employed the Brown (1974) shear-layer-growth model (without the factor of 2 in the denominator).

We note that the data of Sirieix \& Solignac (1966) are normalized at $M_{c, \text { ref }}=1.125$, the lowest compressibility reported. The same $C_{\delta}$ was used for the (single-point) data of Ikawa \& Kubota (1975) and for the data of Samimy \& Elliott (1990). No change is made for the (single-point) data of Barre, Quine \& Dussauge (1994), which are based on a thickness estimate yielding a value for $C_{\delta}$ roughly half that of near-full-width measures. The same $C_{\delta}$ was used in normalizing the Slessor (1998) compressible-flow growth-rate data, as in Hall et al. (1993). These experiments were in the same facility and employ the same (visual) growth measure.

\section{REFERENCES}

Barre, S., Quine, C. \& Dussauge, J. P. 1994 Compressibility effects on the structure of mixing layers: Experimental results. J. Fluid Mech. 259, 47-78.

BRown, G. L. 1974 The entrainment and large structure in turbulent mixing layers. Fifth Australasian Conf. on Hydraulics and Fluid Mechanics, pp. 352-359.

Brown, G. L. \& Roshko, A. 1974 On density effects and large structure in turbulent mixing layers. J. Fluid Mech. 64, 775-816.

Chinzei, N., Masua, G., Komuro, T., Murakami, A. \& Kudou, K. 1986 Spreading of two-stream supersonic turbulent mixing layers. Phys. Fluids 29, 1345-1347.

Clemens, N. T. \& Mungal, M. G. 1995 Large-scale structure and entrainment in the supersonic mixing layer. J. Fluid Mech. 284, 171-216.

Dimotakis, P. E. 1986 Two-dimensional shear-layer entrainment. AIAA J. 24, 1791-1796.

Dimotakis, P. E. 1989 Turbulent free shear layer mixing. AIAA Paper 89-0262.

Dimotakis, P. E. 1991a Turbulent free shear layer mixing and combustion. In High Speed Flight Propulsion Systems. Progress in Astronautics and Aeronautics vol. 137, pp. 265-340.

Dimotakis, P. E. $1991 b$ On the convection velocity of turbulent structures in supersonic shear layers. AIAA Paper 91-1724.

Freund, J. B., Moin, P. \& Lele, S. K. 1997 Compressibility effects in a turbulent annular mixing layer. Rep. TF-72. Department of Mechanical Engineering, Stanford University.

Goebel, S. G. \& Dutton, J. C. 1991 Experimental study of compressible turbulent mixing layers. AIAA J. 29, 538-546.

Goebel, S. G., Dutton, J. C., Krier, H. \& Renie, J. P. 1990 Mean and turbulent velocity measurements of supersonic mixing layers. Exps. Fluids 8, 263-272.

Hall, J. L., Dimotakis, P. E. \& Rosemann, H. 1993 Experiments in non-reacting compressible shear layers. AIAA J. 31, 2247-2254.

IKAWA, H. \& KuBOTA, T. 1975 Investigation of supersonic turbulent mixing layer with zero pressure gradient. AIAA J. 13, 566-572.

Kennedy, C. A. \& Chen, J. H. 1998 Mean flow effects on the linear stability of compressible planar jets. Phys. Fluids 10, 615-626.

Kozusko, F., Lasseigne, D. G., Grosch, C. E. \& Jackson, T. L. 1996 The stability of compressible mixing layers in binary gases. Phys. Fluids 8, 1954-1963.

Lagerstrom, P. A. 1964 Laminar flow theory. In Theory of Laminar Flows, High Speed Aerodynamics and Jet Propulsion, vol. 4, pp. 20-285. Princeton University Press.

LU, G. \& LELE, S. K. 1994 On the density ratio effect on the growth rate of a compressible mixing layer. Phys. Fluids 6, 1073-1075.

PAPAMOSCHOU, D. 1986 Experimental investigation of heterogeneous compressible shear layers. PhD thesis, California Institute of Technology. 
Papamoschou, D. 1989 Structure of the compressible turbulent shear layer. AIAA Paper 89-0126.

Papamoschou, D. \& Bunyajitradulya, A. 1996 Evolution of large eddies in compressible shear layers. Phys. Fluids A 9, 756-765.

Papamoschou, D. \& Roshko, A. 1986 Observations of supersonic free shear layers. AIAA Paper 86-0162.

Papamoschou, D. \& Roshko, A. 1988 The compressible turbulent shear layer: an experimental study. J. Fluid Mech. 197, 453-477.

Peroomian, O. \& Kelly R. E. 1996 Effect of density gradients in confined supersonic shear layers. 1. Two-dimensional disturbances. Phys. Fluids 8, 225-240.

Ragab, S. A. \& WU, J. L. 1987 Linear instability waves in supersonic turbulent mixing. AIAA Paper 87-1418.

RAGAB, S. A. \& WU, J. L. 1988 Instabilities in the free shear layer formed by two supersonic streams. AIAA Paper 88-0038.

Ragab, S. A. \& WU, J. L. 1989 Linear instabilities in two-dimensional compressible mixing layers. Phys. Fluids A 1, 957-966.

Samimy, M. \& Elliott, G. S. 1990 Effects of compressibility on the characteristics of free shear layers. AIAA J. 28, 439-445.

Sandham, N. D. \& ReYnolds, W. C. 1989 A numerical investigation of the compressible mixing layer. Stanford Rep. TF-45.

SARKAR, S. 1995 The stabilizing effect of compressibility in turbulent shear flow. J. Fluid Mech. 282, $163-186$.

Sirieix, M. \& SolignaC, J. L. 1966 Contribution a l'etude experimentale de la couche de melange turbulent isobare d'un ecoulment supersonique. In Separated Flows, AGARD CP4.

SLessor, M. D. 1998 Aspects of turbulent-shear-layer dynamics and mixing. PhD thesis, California Institute of Technology.

TAM, C. K. W. \& HU, F. Q. 1989 The instability and acoustic wave modes of supersonic mixing layers inside a rectangular channel. J. Fluid Mech. 203, 51-76.

Vreman, A. W., Sandham, N. D. \& Luo, K. H. 1996 Compressible mixing layer growth rate and turbulence characteristics. J. Fluid Mech. 320, 235-258.

Zeman, O. 1990 Dilatation dissipation: The concept and application in modeling compressible mixing layers. Phys. Fluids A 2, 178-187.

ZHUANG, M. 1990 An investigation of the inviscid spatial instability of compressible mixing layers. $\mathrm{PhD}$ thesis, California Institute of Technology.

Zhuang, M. \& Dimotakis, P. E. 1995 Instability of wake-dominated compressible mixing layers. Phys. Fluids 7, 2489-2495.

Zhuang, M., Dimotakis, P. E. \& Kubota, T. $1990 a$ The effect of walls on a spatially growing supersonic shear layer. Phys. Fluids A 2, 599-604.

Zhuang, M., Kubota, T. \& Dimotakis, P. E. $1990 b$ Instability of inviscid, compressible free shear layers. AIAA J. 28, 1728-1733. 